TITLE:

\title{
Numerical Analysis of lo's Atmospheric Behavior during Eclipse Based on a Model Boltzmann Equation
}

\author{
$\operatorname{AUTHOR}(\mathrm{S})$ : \\ Kosuge, Shingo; Aoki, Kazuo
}

\section{CITATION:}

Kosuge, Shingo ...[et al]. Numerical Analysis of Io's Atmospheric Behavior during Eclipse Based on a Model Boltzmann Equation. AIP Conference Proceedings: 28th International Symposium on Rarefied Gas Dynamics 2012 2012, 1501: 1541-1548

ISSUE DATE:

2012-11-27

URL:

http://hdl.handle.net/2433/187985

\section{RIGHT:}

(C) 2012 American Institute of Physics. This article may be downloaded for personal use only. Any other use requires prior permission of the author and the American Institute of Physics. 


\title{
Numerical Analysis of Io's Atmospheric Behavior during Eclipse Based on a Model Boltzmann Equation
}

\author{
Shingo Kosuge and Kazuo Aoki \\ Department of Mechanical Engineering and Science, and Advanced Research Institute of Fluid Science and \\ Engineering, Graduate School of Engineering, Kyoto University, Kyoto 606-8501, Japan
}

\begin{abstract}
Unsteady behavior of Io's atmosphere caused by condensation and sublimation of $\mathrm{SO}_{2}$ (the main atmospheric component) during and after eclipse is studied on the basis of kinetic theory. A deterministic computation for a model Boltzmann equation by means of a finite-difference method is performed to obtain the time evolution of the profiles of macroscopic quantities (density, flow velocity, and temperature) in high resolution. As a result, the transient wave motion and oscillatory behavior in the profiles are clarified. To concentrate on the dominant effect of the noncondensable gas (SO or $\mathrm{O}_{2}$ ), other effects in the real atmosphere (e.g., plasma impingement, chemical reactions, etc.) are all omitted in the present analysis. Despite those simplifications, the overall behavior of the atmospheric column is similar to the more realistic result of the previous DSMC analysis in C. H. Moore et al., Icarus 201, 585 (2009).
\end{abstract}

Keywords: Io atmosphere, sublimation, eclipse, Boltzmann equation, rarefied gas dynamics

PACS: $96.30 .1 b, 96.25 . \mathrm{Fx}, 47.45 .-\mathrm{n}, 51.10 .+\mathrm{y}, 64.70 . \mathrm{Hz}$

\section{INTRODUCTION}

The dynamics of Io's atmosphere under sublimation and condensation of its main component, sulfur dioxide $\left(\mathrm{SO}_{2}\right)$ gas, has been studied for a long time (see, e.g., Refs. [1, 2, 3] and references therein). In Ref. [4], unsteady onedimensional behavior of an atmospheric column during eclipse was investigated for the first time via the DSMC simulation of the Boltzmann equation. The results in Ref. [4] clearly show the effect of minor components $\left(\mathrm{SO}_{\text {or }} \mathrm{O}_{2}\right)$, which are considered to be (partially) noncondensable, as a barrier to the atmospheric collapse during eclipse. A trace of noncondensable gas is carried by the condensing flow of $\mathrm{SO}_{2}$ and accumulates on the surface at the early stage of eclipse. Then the accumulated noncondensable gas hinders further condensation of $\mathrm{SO}_{2}$ and significantly delays the atmospheric collapse.

The DSMC analysis in Ref. [4] was extended in subsequent studies [5, 6, 7] by including various real effects, such as three dimensionality, plasma-induced chemistry, electric and magnetic fields, sophisticated surface model, and so on. Those extensions are surely important in, e.g., comparing the simulation with observation results. On the other hand, the complexity may obscure the key physical and gasdynamical effect in the atmospheric behavior concerned.

In the present study (and our recent paper [8]), we consider essentially the same problem as in Ref. [4] after making further simplifications; that is, to concentrate on the dominant effect of the noncondensable gas, other effects included in Ref. [4] (i.e., plasma impingement, molecular internal structure, etc.) are all omitted. In addition, we employ the model Boltzmann equation proposed in Ref. [9] for computational convenience. However, we perform an accurate finite-difference analysis and obtain detailed time evolution of the profiles of physical quantities free from stochastic noise. The solutions with high temporal and spatial resolution reveal some new phenomena, which were not noticed in the previous DSMC analysis [4], and thus complement the results in Ref. [4].

\section{PROBLEM AND ASSUMPTIONS}

Consider a fixed point on Io's equator and in the sub-Jovian hemisphere. The ground is located at $X_{1}=0$ and is covered by the frost of $\mathrm{SO}_{2}$, where $X$ [= $\left.\left(X_{1}, X_{2}, X_{3}\right)\right]$ be the space rectangular coordinates. The atmosphere extends over the half-space $X_{1}>0$ and is composed of $\mathrm{SO}_{2}$ vapor and another noncondensable gas, $\mathrm{SO}$ or $\mathrm{O}_{2} .{ }^{1}$ The eclipse starts at time

\footnotetext{
1 In reality, SO may be partially condensable in Io's circumstances.
} 
$t=0$ and lasts until $t=120 \mathrm{~min}$. The initial atmosphere is assumed to be in a saturated equilibrium state at rest with uniform temperature $T_{0}$. The surface temperature $T_{w}$, which coincides with $T_{0}$ at $t=0$, varies with time according to the change of insolation [see Eq. (11) below] and then condensation or sublimation of $\mathrm{SO}_{2}$ may occur. We investigate unsteady one-dimensional behavior of the atmospheric column over the fixed point during eclipse under the following assumptions: (i) the behavior of the atmosphere is described by the model Boltzmann equation for mixtures proposed in Ref. [9]; (ii) the vapor ( $\mathrm{SO}_{2}$ gas) obeys the complete-condensation boundary condition on the surface [see Eq. (7) below]; (iii) the noncondensable gas $\left(\mathrm{SO}\right.$ or $\left.\mathrm{O}_{2}\right)$ obeys the diffuse-reflection boundary condition on the surface.

\section{FORMULATION}

In the following, the vapor ( $\mathrm{SO}_{2}$ gas) and noncondensable gas $\left(\mathrm{SO}\right.$ or $\left.\mathrm{O}_{2}\right)$ will be referred to as species $A$ and $B$, respectively. The Greek letters $\alpha$ and $\beta$ will be used to represent the species, i.e., $\alpha, \beta=\{A, B\}$.

Let us denote the velocity distribution function (VDF) of molecules of species $\alpha$ as $F^{\alpha}=F^{\alpha}\left(t, X_{1}, \boldsymbol{\xi}\right)$, where $\boldsymbol{\xi}$ $\left[=\left(\xi_{1}, \xi_{2}, \xi_{3}\right)\right]$ is the molecular velocity. The macroscopic quantities, such as the number density $n^{\alpha}$, flow velocity $\boldsymbol{v}^{\alpha}$ $\left[=\left(v_{1}^{\alpha}, v_{2}^{\alpha}, v_{3}^{\alpha}\right)\right]$, pressure $p^{\alpha}$, and temperature $T^{\alpha}$ of species $\alpha$, are defined as the moments of $F^{\alpha}$ as follows:

$$
n^{\alpha}=\int F^{\alpha} \mathrm{d} \boldsymbol{\xi}, \quad \boldsymbol{v}^{\alpha}=\frac{1}{n^{\alpha}} \int \boldsymbol{\xi} F^{\alpha} \mathrm{d} \boldsymbol{\xi}, \quad p^{\alpha}=k n^{\alpha} T^{\alpha}=\frac{1}{3} \int m^{\alpha}\left|\boldsymbol{\xi}-\boldsymbol{v}^{\alpha}\right|^{2} F^{\alpha} \mathrm{d} \boldsymbol{\xi},
$$

where $m^{\alpha}$ is the molecular mass of species $\alpha, k$ is the Boltzmann constant, and $\mathrm{d} \xi=\mathrm{d} \xi_{1} \mathrm{~d} \xi_{2} \mathrm{~d} \xi_{3}$. The domain of integration is the whole space of $\boldsymbol{\xi}$. The corresponding quantities of the total mixture, i.e., the number density $n$, flow velocity $\boldsymbol{v}\left[=\left(v_{1}, v_{2}, v_{3}\right)\right]$, pressure $p$, and temperature $T$ of the mixture, are given by

$$
n=\sum_{\alpha=A, B} n^{\alpha}, \quad \boldsymbol{v}=\sum_{\alpha=A, B} m^{\alpha} n^{\alpha} \boldsymbol{v}^{\alpha} / \sum_{\alpha=A, B} m^{\alpha} n^{\alpha}, \quad p=k n T=\sum_{\alpha=A, B}\left(p^{\alpha}+\frac{1}{3} m^{\alpha} n^{\alpha}\left|\boldsymbol{v}^{\alpha}-\boldsymbol{v}\right|^{2}\right) .
$$

Note that the horizontal components of the flow velocity will be ignored (i.e., $v_{2}^{\alpha}=v_{3}^{\alpha}=v_{2}=v_{3}=0$ ) in the actual analysis, whereas they are left in the formulation.

\section{Model Boltzmann Equation}

The model Boltzmann equation in Ref. [9] for the present problem may be written as follows:

$$
\frac{\partial F^{\alpha}}{\partial t}+\xi_{1} \frac{\partial F^{\alpha}}{\partial X_{1}}-g \frac{\partial F^{\alpha}}{\partial \xi_{1}}=K^{\alpha}\left(M^{\alpha}-F^{\alpha}\right), \quad(\alpha=A, B) .
$$

Here, $g\left(=1.8 \mathrm{~m} / \mathrm{s}^{2}\right)$ is the gravitational acceleration on Io, which is treated as a constant since the scale height of the atmosphere is much smaller than Io's radius. The $K^{\alpha}$ and $M^{\alpha}$ are defined by

$$
K^{\alpha}=\sum_{\beta=A, B} K^{\beta \alpha} n^{\beta}, \quad M^{\alpha}=n^{\alpha}\left(\frac{m^{\alpha}}{2 \pi k T^{(\alpha)}}\right)^{3 / 2} \exp \left(-\frac{m^{\alpha}\left|\boldsymbol{\xi}-\boldsymbol{v}^{(\alpha)}\right|^{2}}{2 k T^{(\alpha)}}\right) .
$$

The $K^{\beta \alpha}\left(=K^{\alpha \beta}\right)$ is a positive constant, that determines the collision frequency of an $\alpha$-species molecule with $\beta$ species molecules via $K^{\beta \alpha} n^{\beta}$. Thus, the above $K^{\alpha}$ corresponds to the total collision frequency of an $\alpha$ molecule. The velocity $\boldsymbol{v}^{(\alpha)}$ and temperature $T^{(\alpha)}$ of the Maxwellian $M^{\alpha}$ are defined by

$$
\begin{aligned}
& \boldsymbol{v}^{(\alpha)}=\boldsymbol{v}^{\alpha}+\frac{2}{m^{\alpha} K^{\alpha}} \sum_{\beta=A, B} \mu^{\beta \alpha} \Omega^{\beta \alpha} n^{\beta}\left(\boldsymbol{v}^{\beta}-\boldsymbol{v}^{\alpha}\right), \\
& T^{(\alpha)}=T^{\alpha}-\frac{m^{\alpha}}{3 k}\left|\boldsymbol{v}^{(\alpha)}-\boldsymbol{v}^{\alpha}\right|^{2}+\frac{4}{K^{\alpha}} \sum_{\beta=A, B} \frac{\mu^{\beta \alpha} \Omega^{\beta \alpha} n^{\beta}}{m^{\beta}+m^{\alpha}}\left(T^{\beta}-T^{\alpha}+\frac{m^{\beta}}{3 k}\left|\boldsymbol{v}^{\beta}-\boldsymbol{v}^{\alpha}\right|^{2}\right),
\end{aligned}
$$

where $\mu^{\beta \alpha}\left[=m^{\beta} m^{\alpha} /\left(m^{\beta}+m^{\alpha}\right)\right]$ is the reduced mass and $\Omega^{\beta \alpha}\left(=\Omega^{\alpha \beta}\right)$ is an additional positive constant; the positivity of $T^{(\alpha)}$ follows if $\Omega^{\beta \alpha} \leq K^{\beta \alpha}$. Note that Eq. (1) is necessary to complete the model equation because $n^{\alpha}, \boldsymbol{v}^{\alpha}$, and $T^{\alpha}$ appear in Eqs. (4) and (5). 
This model was designed in such a way that, by adjusting $\Omega^{\beta \alpha}$, the momentum and energy exchanges between different species can be the same as those for (pseudo-)Maxwell molecules with an arbitrary value of the angular cutoff parameter. In the present study, however, this property is not used for specifying the value of $\Omega^{\beta \alpha}$. We first specify $K^{A A}$ by the relation

$$
K^{A A}=4 d^{2}\left(\pi k T_{0} / m^{A}\right)^{1 / 2},
$$

where $d\left(=7.16 \times 10^{-10} \mathrm{~m}\right)$ is the nominal diameter of an $\mathrm{SO}_{2}$ molecule. This relation means that the molecular mean free path with respect to $\mathrm{SO}_{2}-\mathrm{SO}_{2}$ collisions in an equilibrium state with temperature $T_{0}$ for the model equation is equal to that for the hard-sphere gas with molecular diameter $d$. Then, for simplicity, $K^{B B}, K^{B A}$, and $\Omega^{B A}$ are all assumed to be identical with $K^{A A}$ [note that $\Omega^{A A}$ and $\Omega^{B B}$ are unnecessary; see Eq. (5)]. Therefore, pseudo-Maxwell behavior of the molecules is not enforced in the present study.

\section{Initial and Boundary Conditions}

The boundary condition on the surface is written as follows. For $X_{1}=0$ and $\xi_{1}>0$,

$$
\begin{aligned}
& F^{\alpha}=n_{w}^{\alpha}\left(\frac{m^{\alpha}}{2 \pi k T_{w}}\right)^{3 / 2} \exp \left(-\frac{m^{\alpha}|\boldsymbol{\xi}|^{2}}{2 k T_{w}}\right), \\
& n_{w}^{A}=p_{w}^{A} / k T_{w}, \quad n_{w}^{B}=-\left(\frac{2 \pi m^{B}}{k T_{w}}\right)^{1 / 2} \int_{\xi_{1}<0} \xi_{1} F^{B} \mathrm{~d} \xi .
\end{aligned}
$$

Here $p_{w}^{A}$ is the saturated vapor pressure of $\mathrm{SO}_{2}$ at temperature $T_{w}$ and is given by the Clausius-Clapeyron relation:

$$
p_{w}^{A}=\Pi \exp \left(-\Gamma / T_{w}\right), \quad\left(\Pi=1.516 \times 10^{13} \mathrm{~Pa} \text { and } \Gamma=4510 \mathrm{~K}\right) .
$$

In the present problem, the variation of $T_{w}$ and corresponding $p_{w}^{A}$ with time would induce the unsteady motion of the atmosphere through the boundary condition (7).

The initial condition is written as follows. At $t=0$,

$$
F^{\alpha}=n_{0}^{\alpha}\left(\frac{m^{\alpha}}{2 \pi k T_{0}}\right)^{3 / 2} \exp \left(-\frac{m^{\alpha}\left(|\boldsymbol{\xi}|^{2}+2 g X_{1}\right)}{2 k T_{0}}\right) .
$$

Here $n_{0}^{\alpha}$ is the initial number density of species $\alpha$ on the surface $\left(X_{1}=0\right) ; n_{0}^{A}=p_{0}^{A} / k T_{0}$ with $p_{0}^{A}$ being the saturated vapor pressure at temperature $T_{0}$ [i.e., $p_{0}^{A}$ is given by Eq. (8) with $T_{w}$ being replaced by $T_{0}$ ]. The initial temperature $T_{0}$ will be chosen in the next section. The concentration $\chi^{B}$ of the noncondensable gas in the initial atmospheric column is written as

$$
\chi^{B}=\int_{0}^{\infty} n^{B}(t=0) \mathrm{d} X_{1} / \int_{0}^{\infty} n(t=0) \mathrm{d} X_{1}=\frac{\left(n_{0}^{B} / m^{B}\right)}{\left(n_{0}^{A} / m^{A}\right)+\left(n_{0}^{B} / m^{B}\right)} .
$$

In the following, the amount of the noncondensable gas will be specified by $\chi^{B}$, instead of $n_{0}^{B}$.

\section{Surface Temperature}

The surface temperature $T_{w}$ is determined by the same differential equation as that in Ref. [4]:

$$
\frac{\mathrm{d} T_{w}}{\mathrm{~d} t}= \begin{cases}A \sigma\left(T_{\mathrm{Min}}^{4}-T_{w}^{4}\right), & \text { for } 0 \leq t \leq 120 \mathrm{~min} \\ A \sigma\left(T_{\mathrm{E}}^{4}-T_{w}^{4}\right), & \text { for } t>120 \mathrm{~min}\end{cases}
$$

where $\sigma$ is the Stefan-Boltzmann constant and $A=\varepsilon / C$ with $\varepsilon$ being the bolometric emissivity and $C$ the heat capacity per unit area of the surface. The $T_{\mathrm{E}}$ is an equilibrium temperature defined as

$$
T_{\mathrm{E}}= \begin{cases}\left(T_{\mathrm{Max}}-T_{\mathrm{Min}}\right) \cos ^{1 / 4} \theta+T_{\mathrm{Min}}, & \text { for } \theta \leq 90^{\circ}, \\ T_{\mathrm{Min}}, & \text { for } \theta>90^{\circ},\end{cases}
$$


TABLE 1. Simulation cases.

\begin{tabular}{ccccc|ccccc}
\hline Case & $T_{0}(\mathrm{~K})$ & Longitude $\left(^{\circ}\right)$ & $\operatorname{Gas} B\left(\chi^{B}\right)$ & $A^{-1}\left(\mathrm{~J} / \mathrm{m}^{2} \mathrm{~K}\right)$ & Case & $T_{0}(\mathrm{~K})$ & Longitude $\left(^{\circ}\right)$ & $\operatorname{Gas} B\left(\chi^{B}\right)$ & $A^{-1}\left(\mathrm{~J} / \mathrm{m}^{2} \mathrm{~K}\right)$ \\
\hline 1 & 110 & 69 & $-(0)$ & 350 & 6 & 110 & 69 & $\mathrm{O}_{2}(0.07)$ & 350 \\
2 & 110 & 69 & $\mathrm{SO}(0.35)$ & 350 & 7 & 115 & 52 & $-(0)$ & 350 \\
3 & 110 & 69 & $\mathrm{SO}(0.35)$ & 700 & 8 & 115 & 52 & $\mathrm{SO}(0.22)$ & 350 \\
4 & 110 & 69 & $\mathrm{SO}(0.35)$ & 175 & 9 & 120 & 351 & $-(0)$ & 350 \\
5 & 110 & 69 & $\mathrm{O}_{2}(0.35)$ & 350 & 10 & 120 & 351 & $\mathrm{SO}(0.03)$ & 350 \\
\hline
\end{tabular}

where $\theta$ is the solar zenith angle (SZA), which varies with time according to the diurnal motion of the sun. The maximum and minimum of $T_{\mathrm{E}}$ are fixed as $T_{\mathrm{Max}}=120 \mathrm{~K}$ and $T_{\mathrm{Min}}=90 \mathrm{~K}$ throughout the following analysis.

The initial temperature $T_{0}$ appearing in Eq. (9) is chosen as $T_{0}=T_{\mathrm{E}}(t=0)$ using Eq. (12), after we specify the location of the atmospheric column (or the longitude on the equator) and calculate the SZA $\theta$ as a function of time $t$ (note that $t=0$ is defined to be the time of ingress into eclipse). It should be noted that the above $T_{w}$ is influenced only by the insolation and not by the atmospheric behavior (i.e., not by the latent heat and sensible heat from the gas), since the former is dominant. We solve Eq. (11) with the initial condition $T_{w}(t=0)=T_{0}$ to obtain $T_{w}(t)$ beforehand.

\section{NUMERICAL ANALYSIS}

We first eliminate the molecular-velocity variables $\xi_{2}$ and $\xi_{3}$ from the initial-boundary value problem (3), (7), and (9) by introducing appropriate marginal VDF's. Then, the reduced problem with three independent variables $t, X_{1}$, and $\xi_{1}$ is solved by a finite-difference method. In the present study, we used the following two schemes: (i) the same implicit scheme as in Ref. [8], where the derivatives with respect to $X_{1}$ and $\xi_{1}$ are expressed by the 2nd-order up-wind finitedifference; (ii) 2nd-order Runge-Kutta (Heun's) scheme along the characteristics of Eq. (3) in combination with the interpolation method devised in Ref. [10]. In the latter scheme, because of the properties of the method in Ref. [10], the transient waves tend to be more accurately captured without overshoots in the profiles of the macroscopic quantities (and in those of the VDF's). However, as in the cubic interpolated pseudo-particle (CIP) method [11], equations for the derivatives of $F^{\alpha}$ must be solved simultaneously. Thus the latter requires larger amount of computations (and involves some difficulty in the treatment of boundary conditions for the derivatives). To compensate the increased amount of computations, we performed a parallel computing (the latter is an explicit scheme).

The results shown in the next section were obtained by scheme (ii), while the details of the method are omitted here [some test runs with scheme (i) were also performed and gave roughly the same results]. In the computation, we limit the range of $X_{1}$ up to $X_{1} \sim 282-313 \mathrm{~km}$ and impose the specular-reflection condition at the upper boundary ${ }^{2}$; the minimum grid intervals for $T_{0}=110,115$, and $120 \mathrm{~K}$ are, respectively, $15.9 \mathrm{~m}, 8.3 \mathrm{~m}$, and $4.3 \mathrm{~m}$ at $X_{1}=0$; the maximum intervals are about $0.3-1.1 \mathrm{~km}$ at the upper boundary. The range of $\xi_{1}$ is limited as $\left|\xi_{1}\right| \leq 8 c_{0}$, where $c_{0}$ [ $\left.=\left(2 k T_{0} / \mathrm{m}^{A}\right)^{1 / 2}\right]$ is about $173 \mathrm{~m} / \mathrm{s}$ for $T_{0}=115 \mathrm{~K}$; the minimum and maximum grid intervals are $0.005 c_{0}$ at $\xi_{1}=0$ and $0.045 c_{0}$ at $\xi_{1}= \pm 8 c_{0}$, respectively. The time steps are about $4.7 \mathrm{~ms}$ for $T_{0}=110$ and $115 \mathrm{~K}$ and $2.3 \mathrm{~ms}$ for $120 \mathrm{~K}$.

\section{RESULTS}

We consider Cases 1 - 10 listed in Table 1 in the present study. The values of parameters were cited from Ref. [4].

Figure 1 shows the variations of the surface temperature and of the column density of $\mathrm{SO}_{2}$ in the case of $T_{0}=110$ $\mathrm{K}$. The column density of pure $\mathrm{SO}_{2}$ atmosphere (Case 1) decreases significantly at the end of eclipse, whereas in the case of mixtures the decrease is hindered by the noncondensable gas [see Fig. 3(b) below]. The effects of the gas species (i.e., the molecular mass ratio $\left.\mathrm{m}^{B} / \mathrm{m}^{A}\right)$, concentration $\chi^{B}$, and heat capacity of the surface $\left(\sim A^{-1}\right)$ are also examined. Except for some minor differences, the overall behavior of the column shown in Fig. 1 seems to be close to the corresponding result of the previous DSMC analysis (i.e., Fig. 8 in Ref. [4]).

\footnotetext{
2 This condition was used to fix the total amount of the noncondensable gas in the column. A vacuum condition for the vapor, i.e., $F^{A}\left(\xi_{1}<0\right)=0$ at the upper boundary, was also used in some test runs and gave essentially the same results.
} 

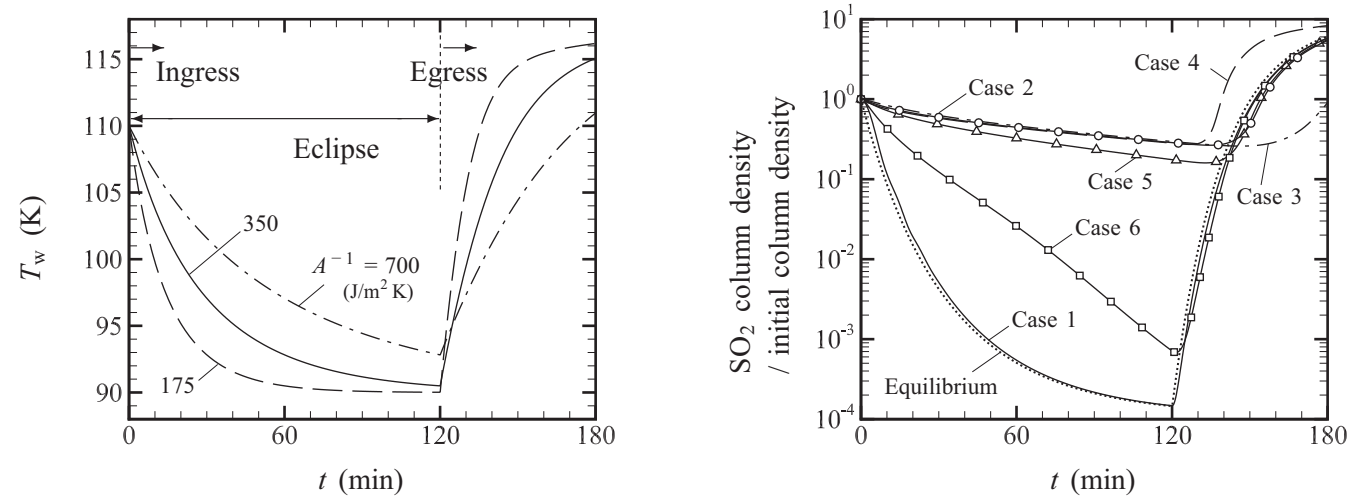

FIGURE 1. Surface Temperature $T_{w}$ and $\mathrm{SO}_{2}$ column density vs. time in the case of $T_{0}=110 \mathrm{~K}$. The initial $\mathrm{SO}_{2}$ column density is $1.2386 \times 10^{20} \mathrm{\#} / \mathrm{m}^{2}$. The dotted line in the right panel indicates the theoretical value for pure $\mathrm{SO}_{2}$ atmosphere in an isothermal saturated equilibrium state at rest when $A^{-1}=350 \mathrm{~J} / \mathrm{m}^{2} \mathrm{~K}$.
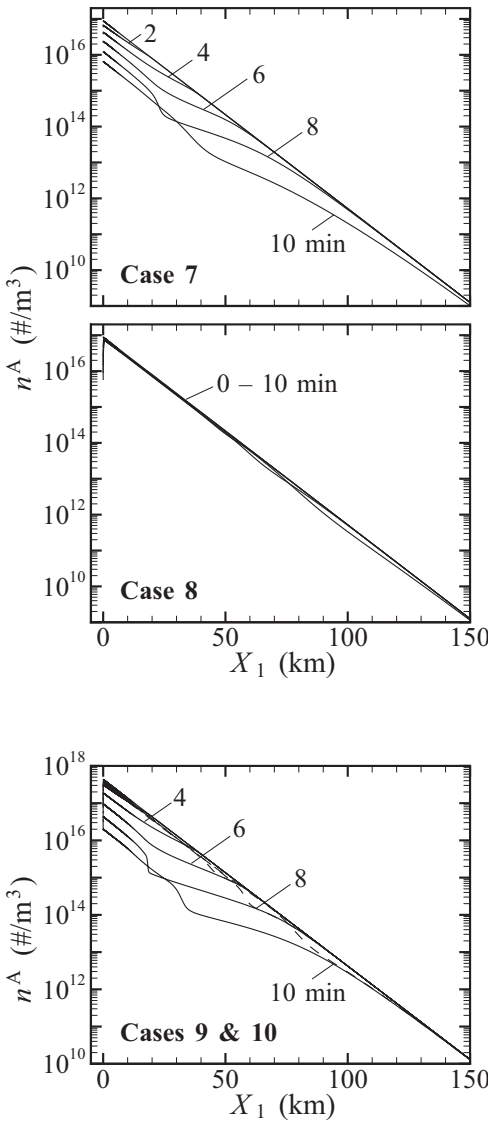
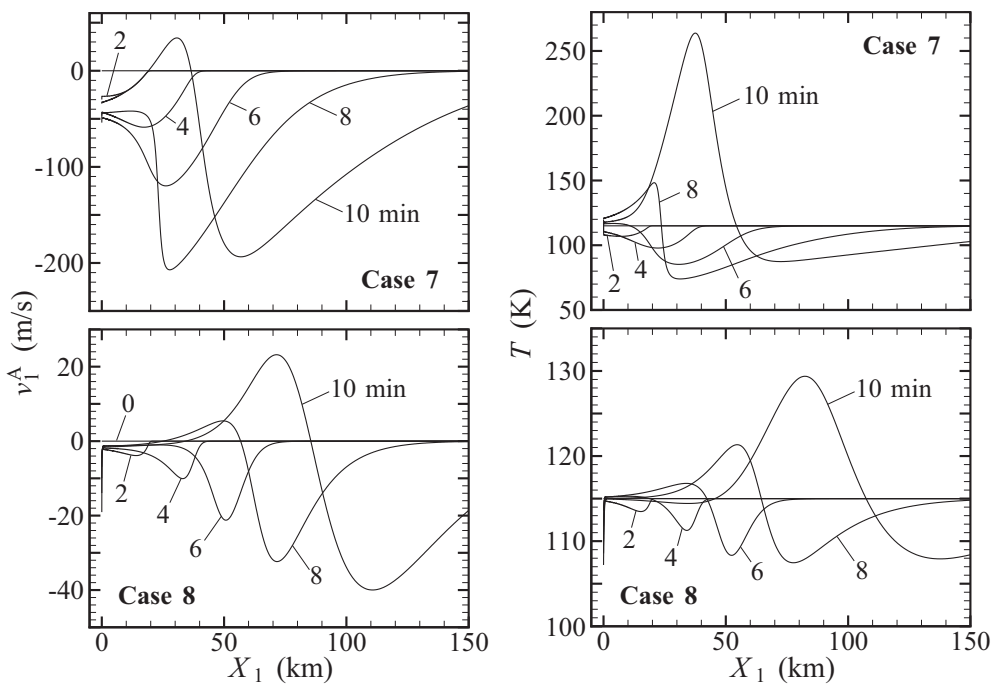

(a)
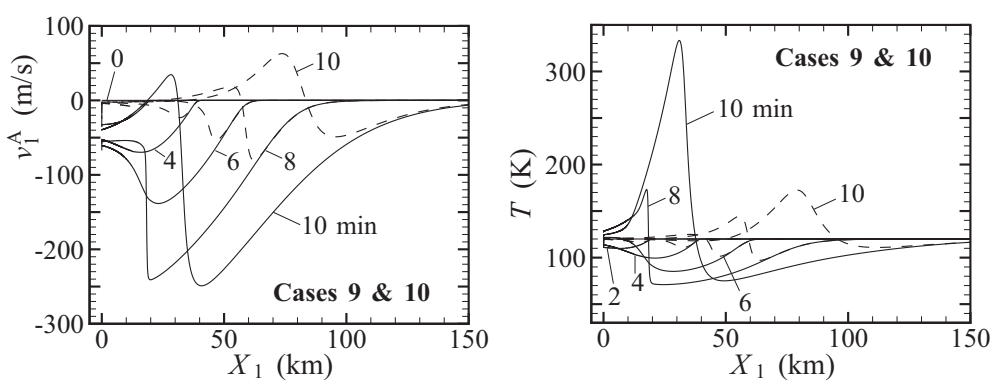

(b)

FIGURE 2. Profiles of the macroscopic quantities at every 2 minutes during the first 10 minutes after ingress. (a) Cases 7 and 8 , and (b) Cases 9 (solid line) and 10 (dashed line). In (b), each of the dashed lines approaches the corresponding solid line for the same $t$ as $X_{1} \rightarrow \infty$. 


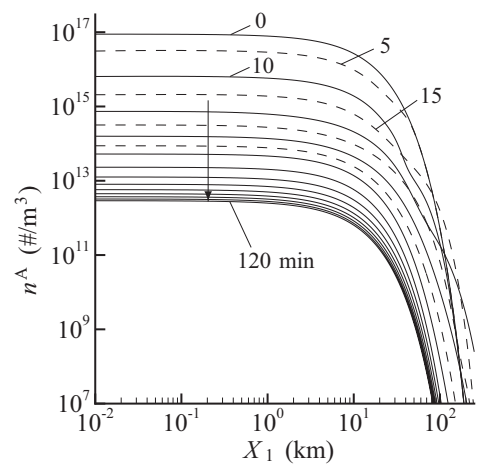

(a) Case 7
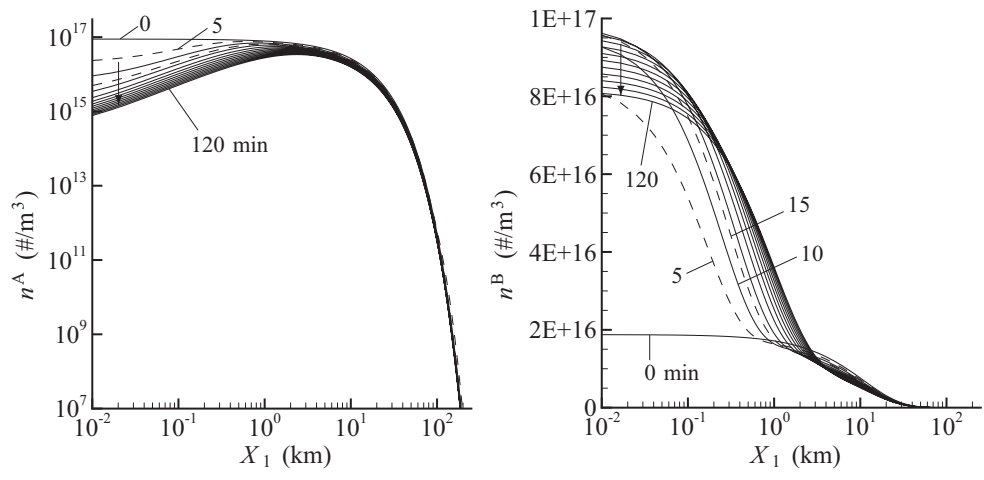

(b) Case 8

FIGURE 3. Number density profiles during eclipse. (a) Case 7 and (b) Case 8 . The solid line indicates profiles at every 10 minutes $(t=0,10, \ldots, 120 \mathrm{~min})$, and the dashed line those at $t=5,15,25$, and $35 \mathrm{~min}$ in (a) and those at $t=5$ and $15 \mathrm{~min}$ in (b).
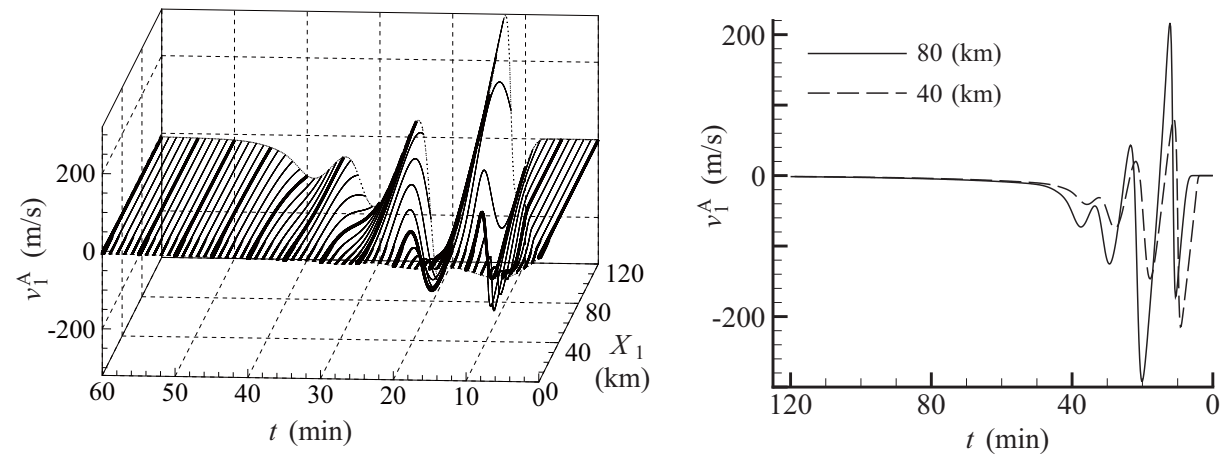

(a) Case 7
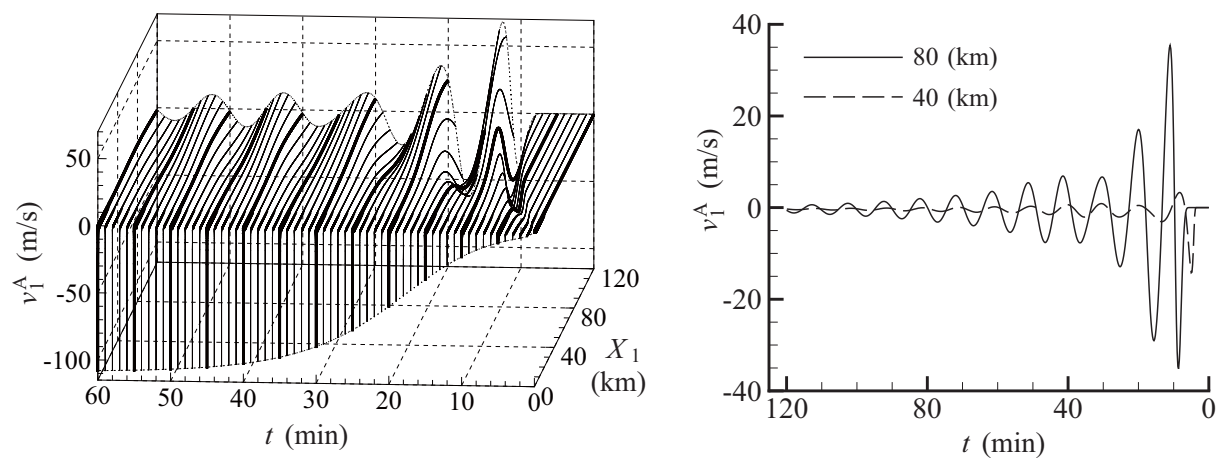

(b) Case 8

FIGURE 4. Profiles of the flow velocity $v_{1}^{A}$ at every minute (left panel) and the cross sections at $X_{1} \simeq 40$ and $80 \mathrm{~km}$ (right panel). (a) Case 7 and (b) Case 8. The thick line in the left panel indicates profiles at every 5 minutes.

\section{During Eclipse}

Figure 2 shows the profiles of macroscopic quantities at the beginning of eclipse. In Cases 7 and 9 (pure $\mathrm{SO}_{2}$ ), a fast condensing flow is induced, and, as a result, an expansion wave is sent upward. The expansion wave is then followed 

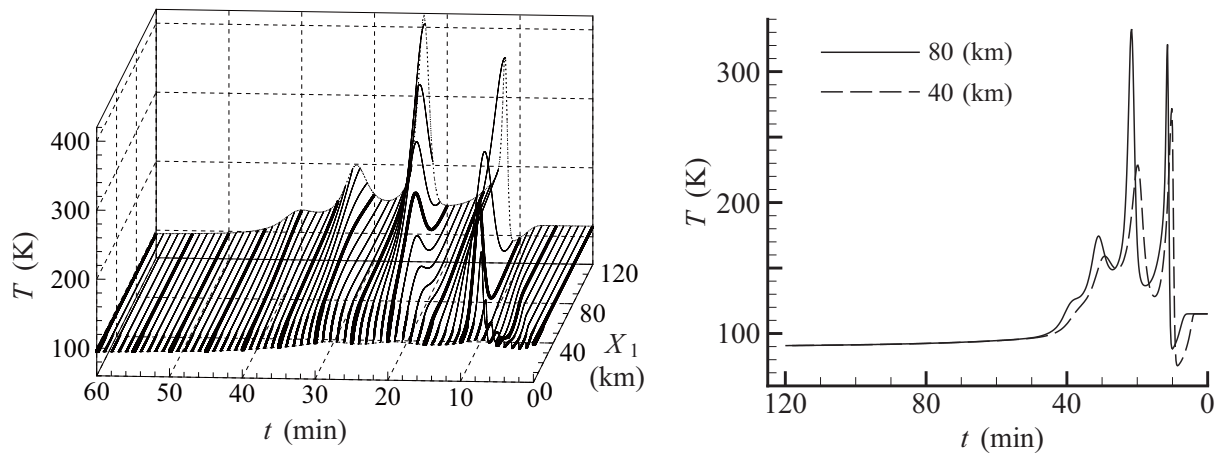

(a) Case 7
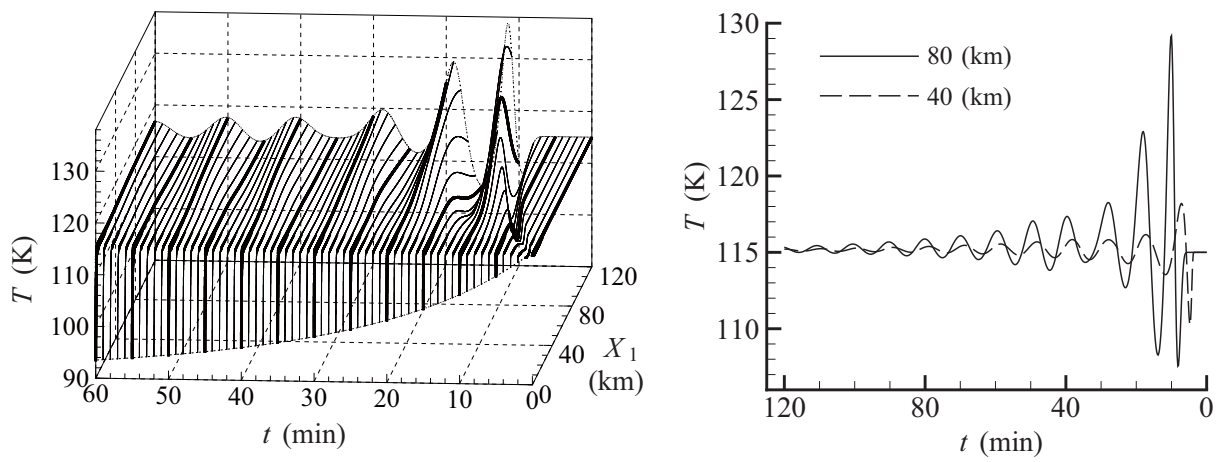

(b) Case 8

FIGURE 5. Profiles of the temperature $T$ at every minute (left panel) and the cross sections at $X_{1} \simeq 40$ and $80 \mathrm{~km}$ (right panel). (a) Case 7 and (b) Case 8 . The thick line in the left panel indicates profiles at every 5 minutes.

by a shock wave appearing near the surface. While propagating upward, the shock wave stretches rapidly because the background pressure decays exponentially with altitude (thus the local mean free path grows exponentially). In Cases 8 and 10 (mixture), the condensing flow is relatively slow because of the hindrance by the noncondensable gas [see Fig. 3(b) below]. The expansion wave is sent as in the pure $\mathrm{SO}_{2}$ case, but is immediately followed by a relatively weak compression wave.

Figure 3 shows the profiles of the number density in Cases 7 and 8 during eclipse. In Case 7 (pure $\mathrm{SO}_{2}$ ), the number density decreases at all altitudes until the end of eclipse except at $t \sim 10-30 \mathrm{~min}$. During that time period, the number density at high altitudes $\left(X_{1} \gtrsim 100 \mathrm{~km}\right)$ is increased temporarily by the passage of the shock wave seen in Fig. 2. In Case 8 (mixture), the number density of $\mathrm{SO}_{2}$ decreases only in the neighborhood of the surface and hardly changes at high altitudes. This is because the noncondensable gas, which is carried by the condensing flow of $\mathrm{SO}_{2}$ to the surface and accumulates there, forms the partial barrier to the atmospheric collapse. The number density of the noncondensable gas near the surface increases rapidly until $t \sim 20 \mathrm{~min}$ and then starts to decrease because of the upward self diffusion.

Figures 4 and 5 show, respectively, the profiles of the flow velocity and temperature in Cases 7 and 8 during eclipse. The oscillatory behavior seen in the figures is produced by waves which, as those in Fig. 2, appear in the lower atmosphere and propagate upward successively. In Case 7, the amplitude of oscillation is large and thus a very fast flow and high temperature may appear instantaneously, especially at high altitudes. The oscillation decays rapidly with time and almost ceases until $t \sim 40 \mathrm{~min}$. In Case 8, while the amplitude is small compared to the pure $\mathrm{SO}_{2}$ case and decays with time, the oscillation continues until the end of eclipse except near the surface. In Case 8, a fast condensing flow in the close vicinity of the surface remains until the end of eclipse. This is because the $\mathrm{SO}_{2}$ density on the surface is kept much higher than the saturation density by the effect of the noncondensable gas [see Fig. 3(b)]. The temperature in Case 8 oscillates around the initial temperature $\left(T_{0}=115 \mathrm{~K}\right)$ in most parts of the atmosphere. The atmosphere is cooled only near the surface via conduction. 


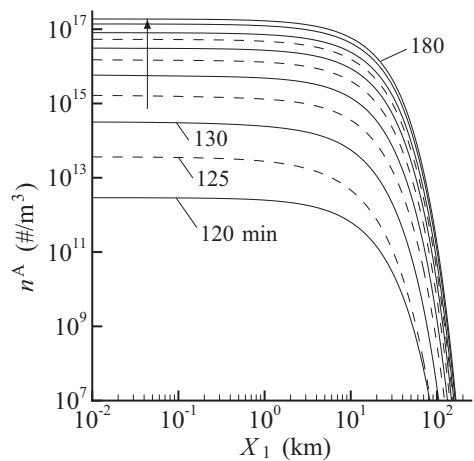

(a) Case 7
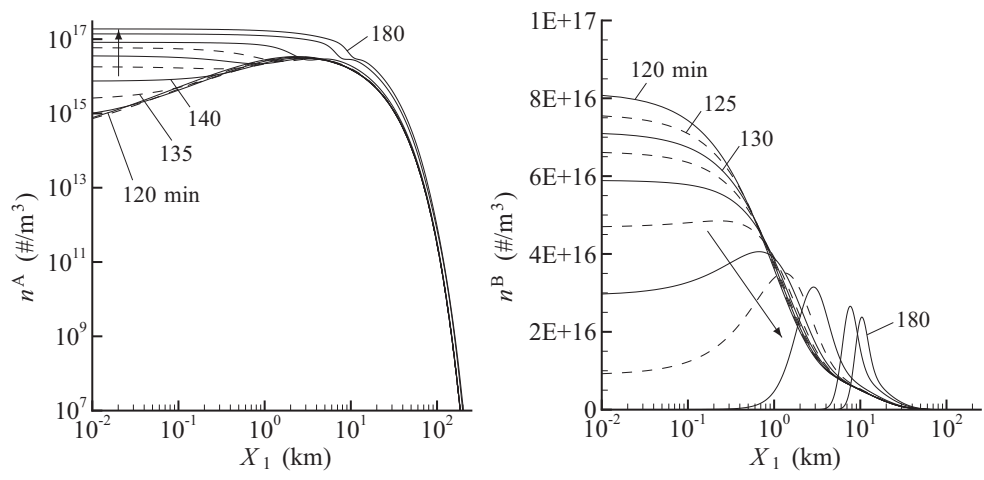

(b) Case 8

FIGURE 6. Number density profiles after egress. (a) Case 7 and (b) Case 8 . The solid line indicates profiles at every 10 minutes $(t=120,130, \ldots, 180 \mathrm{~min})$, and the dashed line those at $t=125,135,145$, and $155 \mathrm{~min}$.

\section{After Egress}

Figure 6 shows the profiles of the number density in Cases 7 and 8 after egress. In Case 7, the number density starts to increase immediately after egress and the initial density on the surface is restored at $t \sim 160 \mathrm{~min}$. In Case 8 , the number density of $\mathrm{SO}_{2}$ remains almost unchanged during the first 10 minutes after egress until the surface temperature and the corresponding saturation density increase sufficiently and the sublimation starts. The noncondensable gas is swept upward by the sublimating flow of $\mathrm{SO}_{2}$ and forms a layer centered around $X_{1}=10 \mathrm{~km}$ at $t=180 \mathrm{~min}$. Correspondingly, a hollow is seen in the profile of $\mathrm{SO}_{2}$ density.

\section{ACKNOWLEDGMENTS}

This work was partially supported by the Grants-in-Aid for Scientific Research, No. 23246034 and No. 23360048 from JSPS.

\section{REFERENCES}

1. A. P. Ingersoll, M. E. Summers, and S. G. Schlipf, Icarus 64, 375-390 (1985).

2. J. V. Austin and D. B. Goldstein, Icarus 148, 370-383 (2000).

3. W. H. Smyth and M. C. Wong, Icarus 171, 171-182 (2004).

4. C. H. Moore, D. B. Goldstein, P. L. Varghese, L. M. Trafton, and B. Stewart, Icarus 201, 585-597 (2009).

5. A. C. Walker, S. L. Gratiy, D. B. Goldstein, C. H. Moore, P. L. Varghese, L. M. Trafton, D. A. Levin, and B. Stewart, Icarus 207, 409-432 (2010).

6. A. C. Walker, Ph.D thesis, University of Texas at Austin (2012).

7. A. C. Walker, C. H. Moore, D. B. Goldstein, P. L. Varghese, and L. M. Trafton, Icarus, (submitted).

8. S. Kosuge, K. Aoki, T. Inoue, D. B. Goldstein, and P. L. Varghese, Icarus, (submitted).

9. P. Andries, K. Aoki, and B. Perthame, J. Stat. Phys. 106, 993-1018 (2002).

10. F. Xiao, T. Yabe, G. Nizam, and T. Ito, Comput. Phys. Commun. 94, 103-118 (1996).

11. H. Takewaki, A. Nishiguchi, and T. Yabe, J. Comput. Phys. 61, 261-268 (1985). 
Copyright of AIP Conference Proceedings is the property of American Institute of Physics and its content may not be copied or emailed to multiple sites or posted to a listserv without the copyright holder's express written permission. However, users may print, download, or email articles for individual use. 